\title{
PRÁTICAS DE ENVOLVIMENTO PRODUZEM COLABORADORES RESILIENTES? MEDIAÇÃO DA IDENTIDADE ORGANIZACIONAL
}

\section{INVOLVEMENT PRACTICES PRODUCE RESILIENT EMPLOYEES? MEDIATION OF ORGANIZATIONAL IDENTITY}

Gisela Demo

Universidade de Brasília - Brasil

giselademo@gmail.com

Letícia Fenelon

Universidade de Brasília - Brasil leticia_fenelon@hotmail.com

Ana Carolina Costa

Universidade de Brasília - Brasil

carolrezendecosta@gmail.com
Eluiza Alberto de Morais Watanabe Universidade de Brasília - Brasil

eluizaw@gmail.com

Submissão: 16 Set 2018. Aceitação: 01/09/2019.

Publicação: 30 Set. 2019. Sistema de avaliação: Double

blind review. Universidade FUMEC / FACE, Belo Horizonte

- MG, Brasil. Editores: Prof. Dr. Mário Teixeira Reis Neto e

Prof. Dr Cid Gonçalves Filho

\section{RESUMO}

A Gestão de Pessoas (GP) assume importância estratégica para a gestão dos colaboradores de uma organização. A cultura corporativa, em parte constituída pelas práticas de GP, tem relação com a identidade organizacional, que está ligada ao alcance dos objetivos organizacionais. Outrossim, as organizações passaram a demandar que seus colaboradores fossem resilientes. Assim, essa pesquisa, de natureza quantitativa, tem como objetivo avaliar a relação entre as práticas de envolvimento, a identidade organizacional e resiliência no trabaIho de empregados públicos. Como resultados, a resiliência no trabalho é uma consequente da percepção das práticas de envolvimento e possui a identidade organizacional como variável mediadora na relação. Esta pesquisa avança ao se debruçar sobre um modelo ainda inexplorado na literatura científica e aponta para a necessidade de um conjunto de práticas de envolvimento bem desenvolvidas, na medida em que influenciam a identidade organizacional dos trabalhadores, de forma a impactar positivamente sua resiliência.

\section{PALAVRAS-CHAVE}

Práticas de envolvimento. Identidade organizacional. Resiliência no trabalho. Equação estrutural. Mediação. 


\section{ABSTRACT}

Human Resource Management (HRM) assumes strategic importance for the management of the employees of an organization. Corporate culture, in part constituted by HRM practices, is related to organizational identity, which is linked to the achievement of organizational goals. Also, organizations have come to demand that their employees be resilient. Thus, this quantitative research aims to evaluate the relationship between involvement practices, organizational identity and resilience at work of public employees. As results, resilience at work is a consequence of the involvement practices perception and the organizational identity is mediator variable in the relation. This research advances by focusing on an yet unexplored model in the scientific literature and points to the need for a set of well-developed involvement practices insofar as they influence the organizational identity of employees so as to positively impact their resilience.

\section{KEYWORDS}

Involvement practices. Organizational identity. Resilience at work. Structural equation. Mediation.

\section{INTRODUÇÃO}

$\mathrm{Na}$ atual era do conhecimento, em que fatores como tecnologia não são mais considerados suficientes para garantir uma posição competitiva sustentável, as pessoas passam a ser competências essenciais de diferenciação estratégica nas organizações (DEMO, 2016).Assim, a Gestão de Pessoas (GP) assume importância estratégica para a gestão dos colaboradores de uma organização por possuir papel relevante na consecução de metas e tomada de decisões em busca de maior efetividade organizacional (ARMSTRONG, 20I4; HORTA; DEMO; ROURE, 20I2).

Os recursos humanos, no papel de responsáveis pela produção do conhecimento, devem ser valorizados e desenvolvidos por meio de práticas de gestão de pessoas (PGP), as quais definem a abordagem e os princípios em geral sobre questões de gestão de pessoas. Ademais, a GP deve pro- mover um tratamento equitativo entre os colaboradores e auxiliar na construção da cultura corporativa (ARMSTRONG, 20I4).

De acordo com a pesquisa feita por Chen (20II), a cultura corporativa, que é em parte constituída pelas práticas de GP, tem relação positiva com o construto Identidade Organizacional (IO), que por seu turno, está intrinsicamente ligado ao aumento da vantagem competitiva e ao alcance dos objetivos organizacionais. A identidade organizacional pode ser entendida como o vínculo do indivíduo com a organização e com o resultado do senso compartilhado de realidade e interação entre seus membros (MACHADO-DASILVA; NOGUEIRA, 200I; NASCIMENTO; TORRES; SOUZA et al., 20I3).

As organizações, ao mesmo tempo em que passaram a valorizar mais seus colaboradores, também exigiram mais habilidades e características essenciais ao capital 
humano no ambiente de trabalho (DEMO, 2016; RIBEIRO; MATTOS; ANTONELLI et al., 20I I). Outrossim, um dos aspectos do comportamento humano que mais tem sido incentivado na atualidade trata das condições adversas para conviver com a incerteza, ou seja, o estado de resiliência (JOB, 2003; RIBEIRO; MATTOS; ANTONELLI et al., 20I I). Esses autores destacam que a resiliência em contextos organizacionais envolve os fatores de risco - momentos de tensão, estresse e traumas acumulados a partir de fatores pessoais e de ambiente; e de proteção - a habilidade de lutar, a autoestima, o reconhecimento e o apoio das chefias/superiores, em muito estimulados por práticas de gestão de pessoas concernentes à prática de envolvimento (COSTA, 2016).

Pesquisas como as de Nascimento (20I4) e Tataw (20I2) evidenciaram associações positivas entre práticas organizacionais em geral e IO. Especificamente sobre práticas de GP, Bezerra, Demo e Fogaça (2016) também encontraram relações positivas com IO, notadamente com a prática de envolvimento. A prática de envolvimento também foi a principal preditora da resiliência no trabalho (COSTA, 20I6).

Dessa maneira, no presente estudo, dentre as seis grandes práticas propostas por Demo, Neiva, Nunes et al. (20I4), elegeuse a prática de envolvimento para avaliar seu poder de predição sobre a identidade organizacional e a resiliência no trabalho, constituindo um modelo ainda não estudado em meio acadêmico. $O$ lócus da pesquisa foi denominado Banco Box, haja vista que a empresa solicitou anonimato. Em suas metas e compromissos inerentes ao planejamento estratégico estão incluídas diversas ações de gestão de pessoas, e as- sim, optou-se por realizar a pesquisa nessa instituição.

Em vista dos argumentos apresentados e da lacuna identificada na literatura de GP no que tange a relações entre as variáveis em questão e a maior carência de pesquisas na área pública, o presente estudo tem como objetivo avaliar a relação entre as práticas de envolvimento, a identidade organizacional e resiliência no trabalho dos empregados do Banco Box.

\section{FUNDAMENTAÇÃO TEÓRICA PRÁTICAS DE ENVOLVIMENTO}

Atualmente, a gestão de pessoas tem papel de extrema importância para a estratégia das organizações (DEMO, 2016), porém, nos estudos de pesquisadores como Demo (2016), Fischer (1998) e Wood, Tonelli e Cooke (20II), percebe-se que ainda há grande desafio para a GP em agir efetivamente como um agente de mudanças na organização confrontando a cultura de que as atividades de departamento de pessoal são a única atribuição do setor.

Até que se tivesse a perspectiva atual da GP, faltavam as políticas de longo prazo, como a administração de carreiras e a integração com o planejamento estratégico, e a área de GP estava muitas vezes subordinada às áreas administrativa e/ou financeira, distante de uma posição estratégica na organização (DEMO, 20I6). Ao analisar a evolução da GP entre I 980 e 20 I0, o trabalho de Wood, Tonelli e Cooke (20 I I) mostrou que a área estava mais preocupada em alinhar suas políticas ao novo cenário mercadológico cada vez mais competitivo, tendo como um dos principais objetivos a inserção do Brasil nesse contexto.

$\mathrm{Na}$ atual conjuntura de mercado, moderno e globalizado, tornou-se imprescindível 
atrair e capacitar pessoas para que fossem mais eficientes e inovadoras. Dessa forma, a valorização do capital humano passou a ser o principal desafio estratégico da moderna GP, tendo em vista que o processo de gestão de recursos humanos pode ser traduzido no processo de gerenciar talentos humanos para atingir os objetivos organizacionais (BOHLANDER; SNELL, 2009; DEMO, 2016).Assim, as novas perspectivas da atual GP são fundamentadas em práticas com ênfase no empreendimento e nos valores do negócio por meio do envolvimento, integração, participação e desenvolvimento dos recursos mais valiosos para a organização: as pessoas (ARMSTRONG, 20I4; FISCHER, 1998; MASCARENHAS; KIRSHBAUM, 2008).

Outrossim, Legge $(1995 ; 2006)$ dá ênfase a dois pontos: as políticas de GP precisam ser integradas ao plano estratégico e oferecer reforço para uma cultura organizacional apropriada para a empresa; e as pessoas são valiosas fontes de diferencial competitivo. Em harmonia com essas ideias, Barney (199I) defende que o capital humano e suas interações resultam na produção do conhecimento e no alcance das metas da organização, princípios estes que embasam os pressupostos e fundamentos das práticas de GP.

No que tange às nomenclaturas, Demo (2016) conclui que não há consenso quanto aos termos utilizados na literatura quanto a práticas de GP, que podem ser encontradas sob outras denominações, tais quais processos, atividades, sistemas, funções e técnicas de GP.Assim como no estudo da referida autora, a presente pesquisa elegeu o termo "prática" de GP, em consonância também com autores como Dessler (2002), Guest (1987), Legge (1995; 2006) e Mathis e Jackson (2003), com o entendimento de que se trata de uma proposta articulada da organização, com construções teóricas e práticas, para possibilitar o alcance dos objetivos e resultados organizacionais, guiando o pensamento e a ação para a área de GP (DEMO, 2016).

A prática de envolvimento selecionada para o presente estudo teve como base a síntese do estado da arte construído por Demo (2016) e os trabalhos de Demo, Neiva, Nunes et al. (20I2, 20l4) que desenvolveram e validaram a Escala de Políticas e Práticas de Recursos Humanos (EPPRH). Tendo em vista que a presente pesquisa analisou a prática de envolvimento de maneira isolada e não em conjunto com as demais práticas, a EPPRH será utilizada em uma versão reduzida, contendo apenas os itens relativos à prática de envolvimento.

Em consonância com a acepção de prática de envolvimento utilizada na presente pesquisa, a Tabela I apresenta seus principais autores, pontos abordados na literatura e as respectivas definições constitutivas, que é o tipo mais comum em termos de dicionários e teorias (Pasquali, 2010).

Demo, Fogaça, Fernandes et al. (20I5) afirmaram que há uma lacuna na literatura no que tange ao estudo das práticas de envolvimento, tendo em vista que as pesquisas relativas a ela representam aproximadamente apenas $2 \%$ da literatura de PGP existente e com análises focadas mormente na comunicação. Dessa forma, as autoras afirmam que é necessário que estudos futuros procurem investigar também o reconhecimento, relacionamento e participação, pertencentes à prática de envolvimento.Vale ressaltar, ainda, que envolvimento é investigado na maioria dos estudos como atitude do colaborador, entendido como comprometimento, e não 
TABELA 1 - Prática de Envolvimento

\begin{tabular}{|c|c|}
\hline Autores & $\begin{array}{l}\text { Dessler (2002), Dietz, Wikinson e Redman (2010), Mathis e Jackson (2003), Muckinsky (2004), Siqueira } \\
\text { (2008), Sisson (1994), Ulrich, Halbrook, Meder et al. (1991). }\end{array}$ \\
\hline \multirow{13}{*}{$\begin{array}{l}\text { Pontos } \\
\text { abordados } \\
\text { na literatura }\end{array}$} & Impacta na satisfação dos colaboradores e nos resultados corporativos. \\
\hline & Contribui para a efetividade organizacional. \\
\hline & Possibilita o bom uso de habilidades dos empregados, reduzindo desigualdades dentro da organização. \\
\hline & Adaptação dos colaboradores aos seus cargos. \\
\hline & Coerência entre discurso e prática gerencial. \\
\hline & Tratamento respeitoso, atencioso e preocupação com o bem-estar. \\
\hline & Clima de compreensão, cooperação e confiança entre gestores e subordinados e entre colegas. \\
\hline & Integração dos colaboradores. \\
\hline & Estímulo à participação e comunicação constantes. \\
\hline & Autonomia na realização das tarefas e tomada de decisões. \\
\hline & Reconhecimento e feedback contínuos. \\
\hline & Identificação de necessidades, valores e preocupações dos colaboradores, estabelecendo relacionamentos. \\
\hline & Existência de canais de comunicação interna. \\
\hline $\begin{array}{l}\text { Definição } \\
\text { constitutiva }\end{array}$ & $\begin{array}{l}\text { Proposta articulada da organização, com construções teóricas e práticas, para criar um vínculo afetivo com } \\
\text { seus colaboradores, contribuindo para seu bem-estar, em termos de reconhecimento, relacionamento, partici- } \\
\text { pação e comunicação. }\end{array}$ \\
\hline
\end{tabular}

Fonte: adaptado de Demo (2016).

como prática da organização, que é a variável foco dessa pesquisa.

\section{RESILIÊNCIA NO TRABALHO}

No atual contexto de trabalho, espera-se que o colaborador esteja preparado para o enfrentamento dos novos desafios e exigências impostas diariamente no ambiente organizacional (MACHADO, 2003; RIBEIRO; MATTOS; ANTONELLI et al., 20II). Além de ser uma característica exigida pelas organizações, Emílio e Martins (20I2) afirmam que a capacidade de enfrentar situações adversas é um diferencial para que o colaborador se mantenha saudável e com desempenho adequado.

O conceito de resiliência é antigo e inerente ao campo de estudo da física, mas desde o final do século $X X$, passou a ser utilizado nas ciências humanas e, atualmente, é entendido como um processo que permite encarar adversidades e superar crises em indivíduos, grupos e organizações; nesse último contexto, decorre especificamen- te da capacidade individual de atribuir um novo ponto de vista às situações adversas (EMÍLIO; MARTINS, 20I2; GOMIDE; SILVESTRIN; OLIVEIRA, 20I5). Nessa lógica, Batista e Oliveira (2008) complementam que a resiliência tem sido apontada como fator de predição a variáveis de adaptação a adversidades no trabalho, bem-estar e reabilitação pós-traumática.

A compreensão do fenômeno da resiliência no trabalho é de extrema importância para que os gestores possam compreender e desempenhar ações de proteção e prevenção à saúde do colaborador, pois a resiliência em âmbito organizacional permite que a relação entre o indivíduo e seu trabalho se preserve saudável mesmo em um contexto de trabalho globalizado permeado por diversas rupturas (BARLACH; LIMONGI-FRANÇA; MALVEZZI, 2008; RIBEIRO; MATTOS,ANTONELLI et al., 20 I I). Dessa forma, são inerentes à resiliência no trabalho os fatores de risco e de proteção, em conjunto com as estratégicas de 
coping (RIBEIRO; MATTOS, ANTONELLI et al., 20I I). Entre os fatores de risco, que podem ser causadores de doenças, podem ser destacados: a pressão sentida devido a responsabilidades no trabalho, a falta de autonomia, a falta de apoio dos colegas de trabalho e chefia e o assédio moral; já os fatores de proteção abrangem o reconhecimento por parte da chefia e a autonomia e autoestima no trabalho (JOB, 2003), os quais constituem práticas de envolvimento (DEMO, 2016).

Costa (2016) afirma, em seu estudo, que a investigação da resiliência no trabalho está em desenvolvimento na literatura e, embora seja um tema promissor e de grande importância para os gestores, tem-se um número reduzido de pesquisas realizadas, constituindo uma lacuna na literatura científica (EMÍLIO; MARTINS, 20 I2). Nesse sentido, tem-se conhecimento de apenas um instrumento validado em âmbito nacional que seja efetivamente voltado para a mensuração da resiliência no contexto de trabalho, construído por Batista e Oliveira (2008), o qual foi baseado na Escala de Resiliência previamente adaptada e validada por Pesce, Assis, Avanci et al. (2005) e então validado para utilização em contexto organizacional (BATISTA; OLIVEIRA, 20I2; GOMIDE; SILVESTRIN; OLIVEIRA, 20I5).

\section{IDENTIDADE ORGANIZACIONAL}

O conceito de identidade está ligado às características de cada pessoa, pois é o que faz com que se reconheça e seja única, tanto por variáveis internas, como o jeito de se vestir, quanto externas, como as atitudes, e não se restringe apenas ao aspecto individual por abranger também as perspectivas de organizações e grupos sociais (MOINGEON; RAMANANTSOA,
1997). Para Machado (2003), a identidade é resultado de constantes interações; é um construto complexo e resultado de diversas forças, como as crenças, valores e papéis que o indivíduo exerce nos espaços individuais, sociais e do trabalho.

Os sinônimos identidade e identificação são temas constantes nos processos de socialização dos trabalhadores, estratégia e desenvolvimento organizacionais. Dessa forma, a identidade organizacional (IO) é relevante e necessária para o entendimento da ação humana no contexto de trabalho (ASHFORT; HARRISON; CORLEY, 2008; NASCIMENTO; TORRES; SOUZA et al., 20I3). Quanto mais se compreende o construto de IO, maior é a facilidade da empresa em capturar a essência das ações de seus colaboradores. Assim, o conceito se configura como um diferencial para os gestores organizacionais e é essencial para uma gestão eficiente (ASHFORT; HARRISON; CORLEY, 2008; NASCIMENTO; TORRES; SOUZA et al., 20I3).

Machado (2003) afirma que alguns estudos propõem uma abordagem equivocada da $I O$ quando a consideram independente dos âmbitos pessoal, social e do trabalho, comprometendo a correta compreensão do construto, que deve ser estudado observando seus múltiplos níveis de análise. Nesse sentido, para que a presente pesquisa faça uma análise assertiva da identidade em contexto organizacional, serão destacados os níveis pessoal, social e no trabalho em consonância com os estudos de Ashfort, Harrison e Corley (2008), Castells (1999), Fernandes e Zanelli (2006) e Machado (2003).

A identidade pessoal está relacionada a um autoconceito que o indivíduo faz considerando suas próprias características e pode 
ser bem construída quando há um limite psíquico estabelecido entre o que é inerente ao individual e o que é resultado dos grupos com os quais a pessoa possui vínculo. Essa identidade envolve um processo de comparação de igualdades e semelhanças entre os indivíduos que envolvem o questionamento: "Quem sou eu?" (ASHFORT; HARRISON; CORLEY, 2008; MACHADO, 2003; PRATT; FOREMAN, 2000).

Em contrapartida, a identidade social pode ser considerada um processo dinâmico construído por semelhanças e diferenças que resultam da interação do autoconceito e do senso de pertencimento, constituído de modo cognitivo e coletivo por critérios individuais, a um ou vários grupos e remete à problemática: "Quem somos nós?" (BEZERRA; DEMO; FOGAÇA, 20 I6; MACHADO, 2003; PRATT; FOREMAN, 2000).

O ambiente de trabalho é uma variável importante a ser destacada quando se trata da construção da identidade, considerando que é um dos principais ambientes em que os indivíduos interagem com pessoas e grupos e constroem ativamente suas identidades, devido a grande parte do tempo em que estão inseridos nesse contexto (MACHADO, 2003; NASCIMENTO; TORRES; SOUZA et al., 20I3). É importante ressaltar, ainda, que a identidade no trabalho se difere da organizacional. A primeira se refere ao vínculo entre o indivíduo e a sua profissão e é em grande parte constituída com base na identificação do funcionário com seus colegas de trabalho, sendo assim processada nos níveis afetivos e cognitivos. A identidade organizacional acontece concomitantemente nesse ambiente, mas está relacionada à ligação psíquica do indivíduo à organização especificamente (MACHA-
DO, 2003; NASCIMENTO;TORRES; SOUZA et al., 20I3).

Considerando os pressupostos de cada nível de identidade, percebe-se que todos estão ligados à representação do eu, do autoconceito para o indivíduo, e acontecem simultaneamente nas esferas pessoais, sociais e no âmbito das organizações, o que contribui para a complexidade do construto (ASHFORT; MAEL, 1989; MACHADO, 2003; PRATT; FOREMAN, 2000). Cabe ainda ressaltar que autores como Machado (2003) e Schultz e Hernes (2013) afirmam o aspecto atemporal do construto, pois consideram a influência do passado na constituição da identidade.

A conceituação de Albert e Whetten (1985) foi elucidativa para a produção do construto $\mathrm{IO}$ e, segundo esses pesquisadores, possui duas formas de ser estudado: a primeira, pelo caráter múltiplo, inferindo que há nas organizações perspectivas compartilhadas pelos colaboradores, grupos e chefias; e a segunda, que considera a identidade organizacional como resultado da visão da alta administração, que acaba por disseminar sua percepção para a empresa como um todo. No presente estudo, será utilizada a primeira forma de compreensão do construto, como expressão do sentimento dos indivíduos quanto à organização em que estão inseridos.

Após a pesquisa inicial deAlbert eWhetten (1985), a identidade organizacional e a sua relevância para sobrevivência e sucesso da organização têm ganhado destaque entre os estudiosos das áreas de administração e psicologia (ALBERT; ASHFORTH; DUTTON, 2000; CORLEY; GIOIA, 2004; NAG; CORLEY; GIOIA, 2007). Estudos também comprovam que a variável pode acarretar uma série de impactos positi- 
vos sobre o desempenho dos funcionários (ASHFORT; MAEL, 1989), dentre eles: a baixa rotatividade, 0 aumento da satisfação e do bem-estar, e o aumento da cidadania organizacional (ASHFORT; HARRISON; CORLEY, 2008; RIKETTA, 2005).

Por conseguinte, Machado (2003) afirmou que a identidade organizacional é construída diariamente e a todo momento a partir das ações e interações dos indivíduos. Portanto, realizar e pertencer são pré-requisitos para que ocorram os processos de identificação e, por consequência, ocorra a transformação do espaço organizacional em um ambiente de trabaIho favorável. Nessa perspectiva, ela exerce uma forte influência sobre a motivação e a produtividade dos trabalhadores, impactando o aumento da vantagem competitiva, a cooperação entre os membros e $o$ alcance dos objetivos organizacionais (CHEN, $20 \mathrm{I}$ I; FERNANDES; ZANELLI, 2006).

Além disso, Fernandes, Marques e Carrieri (2009) afirmaram que os significados atribuídos aos aspectos compartilhados da cultura organizacional delineiam a percepção das pessoas sobre sua identidade organizacional. Adicionalmente, autores como Cuche (1996), Hatch e Schultz (2002) e Prati, Mcmillan-Capehart e Karriker (2009) também afirmam que a cultura organizacional exerce influência sobre a identidade, podendo configurar como variável preditora da IO. Nessa linha de raciocínio, Moingeon e Ramanantsoa (1997) afirmam que um novo funcionário, para ser visto como verdadeiro membro da organização, deverá incorporar os hábitos, crenças, ritos e tabus concernentes a essa, e assim ele estará conectado à empresa e poderá desenvolver sua identidade organizacional.

Nesse contexto em que a instituição possui diversos valores, crenças e princípios compartilhados, a construção das identidades no ambiente de trabalho está vinculada tanto aos interesses pessoais quanto aos coletivos, o que pode resultar em embates por poder e espaço dentro das organizações. Dessa forma, a identidade organizacional figura como fator chave para a motivação e construção de um ambiente saudável que promova a integração entre os funcionários (MACHADO, 2003).

Quanto a pesquisas recentes, foram encontradas na literatura os estudos de Nascimento, Torres, Souza et al. (20l3), que pesquisaram a influência de práticas organizacionais no geral sobre a 10 e o de Bezerra, Demo e Fogaça (2016) que investigaram o papel das práticas de gestão de pessoas na construção da identidade organizacional no setor privado. Entre os resultados encontrados, verificou-se que as dimensões Envolvimento e Recrutamento e Seleção são preditoras de Identidade Organizacional.

Além disso, há estudos que certificam a existência de reflexos positivos da identidade organizacional sobre outros fatores como retenção de funcionários, comportamento criativo, orientação para o cliente, melhoramento de processos em times virtuais, aumento da cooperação mútua em situações de estresse no trabalho e defesa da organização (MAEL;ASHFORT, 1992; TYLER, (999).

Tais fatores podem estar relacionados também ao construto da resiliência no trabalho, uma vez que empresas com empregados mais resilientes geralmente possuem menos problemas de rotatividade e de crises geradas por situações de estresse (JOB, 2003; RIBEIRO; MATTOS; ANTONEELI et al., 20I I). Haja vista que a prática de envolvimento também influenciou a resili- 
ência em contexto organizacional (COSTA, 2016), é possível inferir que a identidade organizacional pode ser caracterizada como variável mediadora entre envolvimento e resiliência no trabalho.

\section{MÉTODO}

Quanto aos fins, à natureza, aos meios e ao horizonte temporal, a presente pesquisa se caracteriza como explicativa, quantitativa, survey e transversal, respectivamente. Para estudar a relação entre as variáveis, o modelo de pesquisa adotou como variável preditora ou independente a Prática de Envolvimento, a Identidade Organizacional como variável mediadora e a Resiliência no TrabaIho como variável critério ou dependente.

A organização objeto do estudo, doravante denominada Banco Box (nome fictício utilizado para resguardar o anonimato solicitado), é constituída sob a forma de instituição financeira do governo federal. Dentre as políticas, princípios e diretrizes que guiam a atuação da instituição, sempre estiveram presentes os aspectos que envolvem gestão de pessoas, como aumento do bem-estar e saúde organizacional e aplicação de práticas de gestão de pessoas, principalmente no que tange ao reconhecimento, valorização e treinamento de pessoas e equipes. Em 2016, a organização era composta por aproximadamente 98.000 funcionários, que constituíram a população da presente pesquisa, todos admitidos por concurso público em consonância com a Constituição Federal e com o estatuto da instituição.

A amostra caracterizou-se como não -probabilística e por conveniência, sendo os questionários respondidos presencialmente e de forma voluntária, utilizando-se envelopes individuais e lacrados. Quanto ao tamanho da amostra, foi utilizada a análise de caminhos, via modelagem por equações estruturais, com o método da estimação por máxima verossimilhança (HOYLE, 1995; RAYKOV; MARCOULIDES, 2006; SCHUMACKER; LOMAX, 2004), que é o mais utilizado na análise fatorial confirmatória (AFC) (BROWN, 2006). Assim, é importante selecionar uma amostra mínima que tenha poder estatístico maior que 0,80 (COHEN, 1992), no caso das ciências comportamentais. Considerando os 9 itens concernentes às variáveis com maior número de itens, Prática de Envolvimento e Escala de Identificação Organizacional, e o poder estatístico de $80 \%$, obteve-se a amostra mínima de 114 indivíduos com a utilização do software G-Power 3.I. Em contrapartida, devido à possível perda de sujeitos na etapa de tratamento de dados, foram distribuídos 250 questionários e o retorno obtido foi de 148 respostas.

O questionário utilizado como instrumento de pesquisa foi composto por três escalas já validadas cientificamente e com resultados psicométricos satisfatórios, quais sejam: Escala de Políticas e Práticas de Recursos Humanos (EPPRH), validada por Demo e Rozzett (20I2) e traduzida por Demo, Neiva, Nunes et al. (20 I4), com rho de Jöreskog de 0,92; Escala de Identificação Organizacional (EIO), construída por Mael (1988) e validada por Mael e Ashfort (1992), com rho de Jöreskog de 0,93; e Escala de Resiliência no Trabalho, construída por Batista e Oliveira (2008) e validada para o contexto de trabalho (COSTA, 2016; GOMIDE; SILVESTRIN; OLIVEIRA, 20I5), com rho de Jöreskog de 0,86; e ainda a parte demográfica para caracterização da amostra. Ademais, o instrumento também conteve a carta convite para participação em pesquisa acadêmica, com as orientações 
para o preenchimento do questionário e o termo de consentimento para participação na pesquisa. $O$ instrumento completo foi composto por 35 itens.

Partindo para a etapa de tratamento dos dados, com o auxílio do software SPSS, foi realizada a análise de distribuição de frequências. Posteriormente, utilizou-se o procedimento listwise para a análise de dados faltantes (missing values). Nessa etapa, foram eliminados 7 questionários tidos como não adequados à pesquisa por estarem incompletos. Procedeu-se à investigação quanto à presença de outliers pelo método Mahalanobis (TABACHNICK; FIDELL, 20I3), que identificou 4 outliers. Em seguida, foram analisados os valores de tolerância e os referentes ao fator de inflação de variância, revelando que os dados não apresentaram problemas de multicolinearidade e de singularidade para a amostra pesquisada (MYERS, 1990). Finalmente, foram realizadas análises de pressupostos para a utilização da análise multivariada, quais sejam, homocedasticidade, linearidade e normalidade, utilizando-se gráficos de probabilidade normal e de resíduos (FIELD, 2009; HAIR; BLACK; BABIN et al., 2009). Por fim, a normalidade multivariada (MARÔCO, 20I0) também foi verificada no software AMOS. Como resultado, todos os pressupostos foram devidamente confirmados. Obteve-se, portanto, uma amostra final composta por I 37 sujeitos que atenderam aos critérios estatísticos supracitados.

Em consonância com Sousa e Mendonça (2009), o modelo mediacional explica as diferentes maneiras pelas quais a variável antecedente influencia a variável critério. Esse modelo é caracterizado por uma relação que altera para mais ou para menos a influência da variável antecedente sobre a variável critério. Então, de forma a atestar a condição de mediação da variável $I O$, foram verificadas as quatro condições propostas por Baron e Kenny ( 1986): I) a variável antecedente prediz a variável critério de forma significativa; 2) a variável antecedente prediz a variável mediadora de forma significativa; 3) a mediadora prediz a critério de forma significativa; e 4) na presença da variável antecedente e da mediadora, a relação previamente constatada como significativa entre antecedente e critério diminui ou desaparece.

\section{RESULTADOS E DISCUSSÃO}

Em um primeiro momento, a fim de verificar o ajuste dos modelos de mensuração, a AFC foi realizada com cada variável da pesquisa, Envolvimento, Identidade Organizacional e Resiliência no Trabalho. A estrutura unifatorial da variável latente Envolvimento (9 itens) foi confirmada, assim como a estrutura da Identidade Organizacional (9 itens) e a da Resiliência no TrabaIho (6 itens), conforme Tabela 2. Todos os 24 itens foram significativos ( $p$-valor<0,0 I).

Para verificar o ajuste do modelo, os índices de modificação (I.M.) foram analisados. Os I.M. entre os itens Env.I e Env.2 e entre Res. 19 e Res.24 foram altos. Com isso, uma seta dupla, que indica a correlação, foi inserida entre essas variáveis.

No que diz respeito à ligação entre os itens Env.I e Env.2, segundo Paz (2004), o bem-estar decorre das relações de reciprocidade estabelecidas entre colaborador e organização, o qual reflete o tratamento dispensado por uma parte em relação à outra. Quando as relações entre chefes e subordinados são permeadas pela valorização da competência, da participação dos colaboradores nas decisões e pelo respeito mú- 
TABELA 2 - Variáveis do estudo

\begin{tabular}{|c|c|}
\hline \multicolumn{2}{|r|}{ Envolvimento } \\
\hline Env.1 & A organização onde eu trabalho se preocupa com meu bem-estar. \\
\hline Env.2 & A organização onde eu trabalho me trata com respeito e atenção. \\
\hline Env.3 & A organização onde eu trabalho procura conhecer minhas necessidades e expectativas profissionais. \\
\hline Env.4 & A organização onde eu trabalho estimula a minha participação nas tomadas de decisão e resolução de problemas. \\
\hline Env.5 & $\begin{array}{l}\text { A organização onde eu trabalho reconhece o trabalho que faço e os resultados que apresento (ex: elogios, matérias em } \\
\text { jornais internos, etc.). }\end{array}$ \\
\hline Env.6 & $\begin{array}{l}\text { Na organização onde eu trabalho, os colaboradores e suas chefias desfrutam da troca constante de informações para } \\
\text { o bom desempenho das funções. }\end{array}$ \\
\hline Env.7 & Na organização onde eu trabalho, há um clima de compreensão e confiança dos chefes em relação aos seus colaboradores. \\
\hline Env.8 & Na organização onde eu trabalho, há um clima de confiança e cooperação entre os colegas de trabalho. \\
\hline Env.9 & Na organização onde eu trabalho, há coerência entre discurso e prática gerenciais. \\
\hline \multicolumn{2}{|r|}{ Identidade Organizacional } \\
\hline 10.10 & Quando alguém critica a organização onde eu trabalho é como se estivesse me criticando pessoalmente. \\
\hline IO.11 & Tenho muito interesse no que os outros pensam sobre organização onde eu trabalho. \\
\hline 10.12 & Quando me refiro à organização onde eu trabalho, normalmente falo nós, ao invés de eles. \\
\hline 10.13 & Quando alguém elogia a organização onde eu trabalho é como se estivesse me elogiando. \\
\hline 10.14 & Quando uma matéria na mídia faz crítica à organização onde eu trabalho, eu me sinto constrangido. \\
\hline 10.15 & O sucesso da organização onde eu trabalho é como se fosse o meu sucesso pessoal. \\
\hline \multicolumn{2}{|r|}{ Resiliência no Trabalho } \\
\hline Res.16 & Mantenho interesse no meu trabalho. \\
\hline Res.17 & Quando estou em uma situação difícil, normalmente acho uma saída. \\
\hline Res.18 & Tenho energia suficiente para fazer o que preciso fazer. \\
\hline Res.19 & Sou determinado (a). \\
\hline Res.20 & Posso olhar normalmente para uma situação de diversas maneiras. \\
\hline Res.21 & Meu trabalho tem sentido para mim. \\
\hline Res.22 & Sinto orgulho de ter realizado coisas. \\
\hline Res.23 & Sou uma pessoa com quem as pessoas podem contar em situações de emergência. \\
\hline Res.24 & Sou disciplinado (a). \\
\hline
\end{tabular}

Fonte: elaborada pelas autoras.

tuo, criam-se condições que poderão gerar impactos positivos no trabalhador (MELO, 2004). Assim, comportamentos positivos dos líderes predizem a saúde dos seus liderados (LITTLE; SIMMONS; NELSON, 2007).

No que tange à correlação entre os itens Res. 19 e Res.24, verifica-se na literatura que ambas são características desejadas em líderes, gestores e profissionais em geral, e que estão intrinsecamente associadas. No modelo de proficiência do líder global, desenvolvido por Chong (2008), são definidas doze competências necessárias para o líder global, divididas em quatro dimensões, dentre elas a de gestão de pessoas, que estão relacionadas à performance e à disciplina. $\mathrm{Na}$ análise de Crosby (1991) e Fiorelli (2000), as características de um profissional que faz a diferença passam pela habilidade interpessoal, autoconfiança, determinação, energia, sensibilidade, disciplina, espírito crítico e senso de justiça, sendo interdependentes.

Introduzidas as alterações, os índices de ajuste de cada AFC são mostrados na Tabela 3. As medidas de ajuste absoluto do modelo revelam um CMIN/DF menor do que 5 (SCHUMACKER; LOMAX, 2004). Esse índice representa $\circ X^{2}$, medida fundamental de ajuste (Hair et al., 2009), dividido pelo número de graus de liberdade, a fim de se obter 
um valor de ajuste ao modelo menos sensível ao tamanho da amostra. Já o Comparative Fit Index (CFI) é uma medida de ajuste comparativo e tem amplitude de zero a um, sendo que valores próximos de um são indicativos de bom ajuste (BYRNE, 20 I0). Por fim, o Standardized Root Mean Square Residual (SRMR) é uma medida absoluta que mostra a diferença entre a correlação normalizada observada e a correlação previsível. Seu valor deve ser $<0$, I. A Tabela 3 mostra que todos os índices de ajuste encontrados nas análises foram satisfatórios.

Em seguida, para atingir o objetivo da presente pesquisa, o modelo de caminhos foi construído para testar se a variável Identidade Organizacional medeia a relação entre o Envolvimento e a Resiliência no Trabalho. Em consonância com Baron e Kenny (1986), as relações entre as três variáveis foram testadas. Ou seja, verificou-se a relação direta entre: a) Envolvimento e Resiliência no Trabalho; b) Identidade Organizacional e Resiliência no Trabalho e; c) Envolvimento e Identidade Organizacional. Todas elas foram significativas ( $p$-valor $<0,0$ I).

O próximo passo foi verificar a relação de mediação. $O$ bootstrap, um procedimento recomendado em equações estruturais para testar a mediação, especialmente quando variáveis latentes estão envolvidas (MARÔ$\mathrm{CO}, 20 \mathrm{I0}$ ), foi utilizado.As estimativas levam em conta o parâmetro padronizado da regressão, os erros padronizados, o p-valor e o critical ratio (C.R.). Esse último é obtido pela divisão entre a estimativa não-padronizada do parâmetro e seu erro padrão e, para ser considerado significativo, tem que ser $>|I, 96|$ (BYRNE, 20I0). Ou seja, o Envolvimento afeta positivamente a Identidade Organizacional $\left(\beta=0,25 ; R^{2}=6 \%\right)$ e a Resiliência no Trabalho $(\beta=0,32)$. Por sua vez, a Identidade Organizacional impacta a Resiliência no Trabalho $(\beta=0,34)$. Os resultados são mostrados na Tabela 4 e na Figura $I$. Todos os valores foram significativos.

Além de testar as relações diretas, o efeito indireto do Envolvimento sobre a Resiliência no Trabalho foi calculado e também está mostrado na Figura I.A mediação parcial foi confirmada e o efeito indireto foi significativo $(p$-valor $<0,00)$ e estimado em 0,08 . O coeficiente de regressão $R^{2}$ foi de $27 \%$, ou seja, o Envolvimento e a Identidade Organizacional explicam $27 \%$ da variável dependente, a Resiliência no Trabalho. Essa explicação é considerada de grande efeito, pois foi maior que $26 \%$ (Cohen, 1992). Nota-se, por fim, que os índices de ajuste do modelo foram adequa-

TABELA 3 - Índices de ajuste da AFC

\begin{tabular}{cccc}
\hline Índice de Ajuste & Envolvimento & Identidade Organizacional & Resiliencia no Trabalho \\
\hline CMIN/DF & 3,13 & 2,38 & 1,82 \\
CFI & 0,90 & 0,95 & 0,93 \\
SRMR & 0,06 & 0,05 & 0,06 \\
\hline
\end{tabular}

Fonte: elaborada pelas autoras.

TABELA 4 - Estimativas do Modelo Estrutural

\begin{tabular}{l|c|c|c|c}
\hline & Erro padronizado & C.R. & P-valor & Estimativa \\
\hline Envolvimento $\rightarrow$ Identidade Organizacional & 0,10 & 2,53 & 0,01 & 0,25 \\
\hline Identidade Organizacional $\rightarrow$ Resiliência no Trabalho & 0,06 & 3,28 & 0,00 & 0,34 \\
\hline Envolvimento $\rightarrow$ Resiliência no Trabalho & 0,06 & 3,09 & 0,00 & 0,32 \\
\hline
\end{tabular}

Fonte: elaborada pelas autoras. 


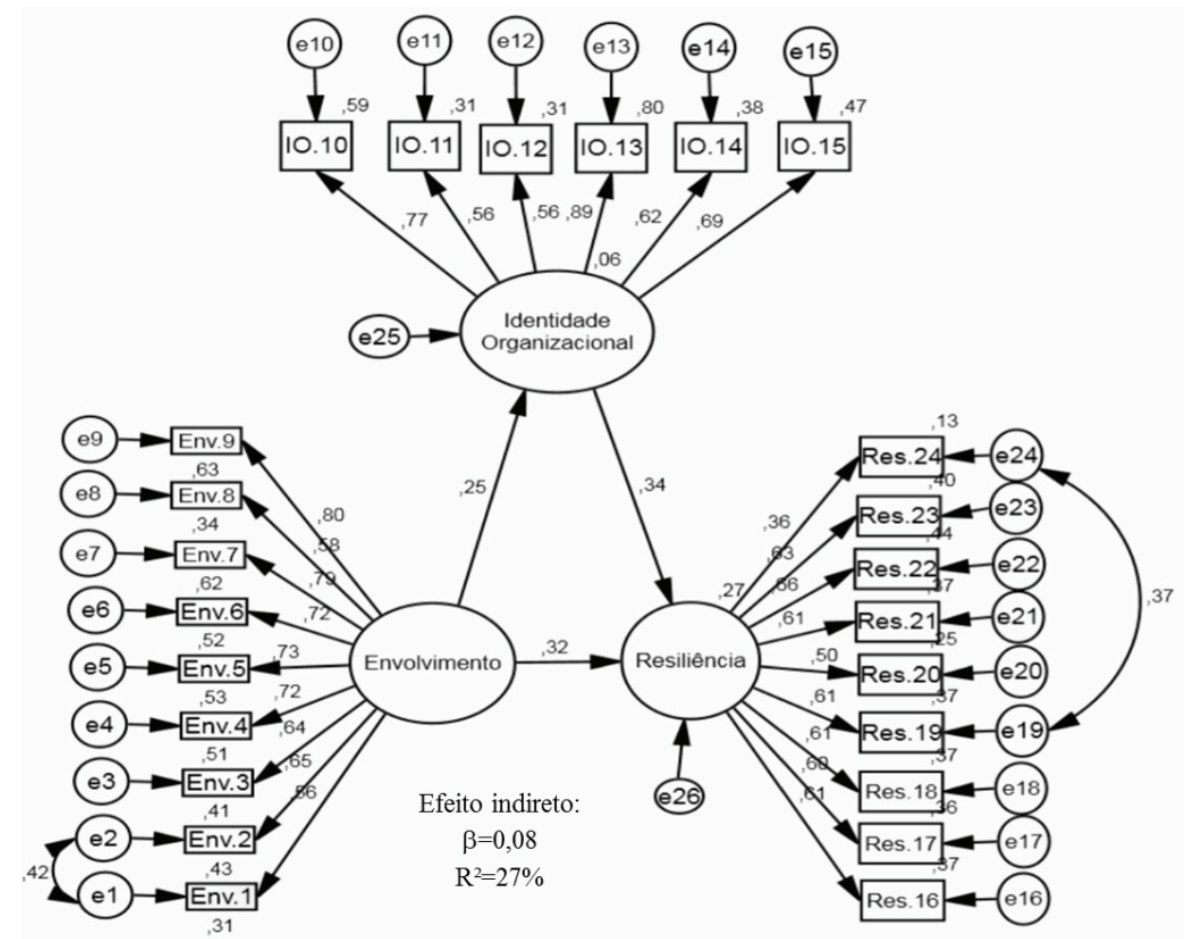

FIGURA 1 - Modelo de Caminhos para testar a mediação. CMIN/DF=1,43; CFI=0,91; SRMR=0,07.

Fonte: elaborada pelas autoras.

dos (CMIN/DF=I,43; CFI=0,9I;SRMR=0,07) confirmando o modelo de mediação hipotetizado na presente pesquisa.

\section{CONCLUSÃO}

Na perspectiva acadêmica, a presente pesquisa contribui para os estudos nas áreas de gestão de pessoas ao abordar três variáveis ainda tratadas de maneira incipiente na literatura. Ademais, Demo, Fogaça e Fernandes et al. (20I5) afirmam que há uma lacuna no que tange ao estudo da prática de envolvimento, haja vista que as pesquisas relativas a ela representam aproximadamente apenas $2 \%$ das pesquisas de PGP. Outrossim, verifica-se que apesar de terem grande importância para os gestores, há um número reduzido de pesquisas realizadas em relação à identidade organizacional e à resiliência no trabalho (ALBERT; ASHFORTH;DUTTON, 2000; COSTA, 2016; EMÍLIO; MARTINS, 20I2).
Adicionalmente, esta pesquisa avança ao se debruçar sobre um modelo ainda inexplorado na literatura científica, com destaque à investigação de mediação, o que também contribui para os estudos organizacionais que pesquisam modelos de antecedentes $\mathrm{e}$ consequentes das variáveis em questão. $\mathrm{Da}$ mesma maneira, o lócus de pesquisa desse estudo também veio somar, uma vez que foi feito em uma empresa pública, diferenciando-se da maioria dos estudos em GP, os quais são realizados no setor privado (DEMO; FOGAÇA; FERNANDES et al., 20I5).

Como sugerem os resultados da pesquisa, a resiliência no trabalho é uma consequente da percepção da prática de envolvimento e possui a identidade organizacional como variável mediadora na relação. Tais considerações apontam para uma necessidade de um conjunto de práticas de envolvimento bem desenvolvidas, na medida em 
que influenciam a identidade organizacional dos trabalhadores, de forma a impactar positivamente a resiliência dos trabalhadores.

Sendo assim, fatores que influenciem a identidade organizacional e a resiliência no trabalho devem ser desenvolvidos/mantidos nas organizações, de forma a se obter um impacto sobre a motivação e a produtividade dos trabalhadores, o aumento da vantagem competitiva, a cooperação entre os membros e o alcance profícuo dos objetivos organizacionais (CHEN, 20I I; FERNANDES; ZANELLI, 2006). Nesse sentido, práticas de envolvimento traduzem um esforço da organização para permitir que a relação entre o indivíduo e seu trabalho se preserve saudável mesmo em um contexto globalizado permeado por elementos estressores, tais quais necessidade de mudanças, pressão por resultados, dentre outros cenários nos quais a resiliência assume conotação especial (BARLACH; LIMONGI-FRANÇA; MALVEZZI, 2008; RIBEIRO; MATTOS, ANTONELLI et al., 20I I).

Dentre as limitações a serem consideradas, está o recorte transversal utilizado nessa pesquisa, uma vez os dados foram coletados em um único momento do tempo e se restringem ao período estudado e à amostra pesquisada, impedindo inferências de generalização e causalidade. Outra limitação refere-se ao fato de o estudo ter sido aplicado em uma única organização.
Dessa forma, recomenda-se estudos comparativos com demais instituições financeiras do setor privado e de economia mista, a fim de se verificar se os resultados encontrados se confirmam para outra amostra de natureza similar, bem como o uso de dados longitudinais.

Considerando o caráter exploratório das relações investigadas, não obstante uma técnica confirmatória de análise tenha sido empreendida, são necessárias novas pesquisas, especialmente multimétodo, para ir além da mensuração e prover uma melhor compreensão de fenômenos parcamente estudados em âmbito nacional, mas relevantes ao bom funcionamento das organizações.

No mesmo sentido, em complementação à agenda de pesquisa proposta por Demo, Fogaça, Fernandes et al. (20I5), sugere-se a realização de mais estudos que investiguem antecedentes e consequentes da prática de envolvimento, avançando para o teste de modelos de mediação ou moderação mais robustos para que haja uma maior consolidação dessa linha de pesquisa.

Por fim, apesar das limitações apresentadas, a presente pesquisa lançou novas possibilidades de pensar o papel de práticas de GP no comportamento organizacional, inaugurando larga avenida para a investigação dos fenômenos, de sorte a propiciar o desenvolvimento de ambientes organizacionais mais saudáveis, resilientes e produtivos. 


\section{REFERÊNCIAS}

ALBERT, S.; ASHFORTH, B. E.; DUTTON, J. E. Organizational identity and identification: charting new waters and building new bridges. Academy of Management Review, v. 25, n. I, p. 13-17, 2000.

ALBERT, S.;WHETTEN, D. A. Organizatoinal identity. In: CUMMINGS, L. L.; STAW, M. M. (Eds.). Research in organizational behavior, v. 7, p. 263-295. Greenwich, CT: JAI, 1985.

ARMSTRONG, M. Armstrong's handbook of human resource management practice. $13 \mathrm{rd}$ ed. London: Kogan Page, 2014.

ASHFORT, B. E.; MAEL, F.A. Social identity theory and the organization. Academy of Management Review, v. 14, n. I, p. 20-39, 1989.

ASHFORT, B. E.; HARRISON, S. H.; CORLEY, K. G. Identification in organizations: an examination of four fundamental questions. Journal of Management, v. 34, n. 3, p. 325-374, 2008.

BARLACH, L.; LIMONGI-FRANÇA, A. C.; MALVEZZI, S. O conceito de resiliência aplicado ao trabalho nas organizações. Revista Interamericana de Psicologia, v. 42, n. I, p. I0I-I I 2, 2008.

BARNEY,J. Firm resources and sustained competitive advantage. Journal of Management, v. I7, n. I, p. 99-120, I99I.

BARON, R. M.; KENNY, D. A. The moderator-mediator variable distinction in social psychological research: Conceptual, strategic and statistical considerations. Journal of Personality and Social Psychology, v. 5I, n. 6, p. II73-II82, 1986.

BATISTA, R. L.; OLIVEIRA,A. de F.Validação da escala de resiliência para o contexto organizacional. In: Congresso Brasileiro de Psicologia Organizacional e do Trabalho,
3, 2008, Santa Catarina. Anais... Santa Catarina: CBPOT, 2008.

BATISTA, R. L.; OLIVEIRA,A. de F.Antecedentes da confiança do empregado na organização. Estudos de Psicologia, v. 17, n. 2, p. 247 254, 2012.

BEZERRA, M. E. A.; DEMO, G.; FOGAÇA, N. O papel das políticas e práticas de gestão de pessoas na construção da identidade organizacional. In: Seminários em Administração da USP, 19, 2016, São Paulo. Anais... São Paulo: SemeAd, 2016.

BOHLANDER, G. W.; SNELL, S. Administração de recursos humanos. I4a ed. São Paulo: Cengage, 2009.

BROWN, T. A. Confirmatory Factor Analysis for Applied Research. Guildford: New York, 2006.

BYRNE, B. M. Structural Equation Modeling with AMOS: basic concepts, applications and programming. Canada: Routledge, 2010.

CASTELLS, M. 0 poder da identidade. São Paulo: Paz e Terra, 1999.

CHEN, Y. S. Green organizational identity: sources and consequence. Management Decision, v. 49, n. 3, p. 384-404, 201 I.

CHONG, E. Managerial competency appraisal: A cross-cultural study of American and East Asian managers. Journal of Business Research, v. 6I, n. 3, p. I91-200, 2008.

COHEN, J.A power primer. Psychological Bulletin, v. II2, n. I, P. I55-159, 1992.

CORLEY, K. G.; GIOIA, D. A. Identity ambiguity and change in the wake of a corporate spin-off. Administrative Science Quarterly, v. 49, n. 2, p. 173-208, 2004.

COSTA, A. C. R. Políticas e práticas de gestão de pessoas pro- duzem servidores públicos resilientes? Validação de um modelo estrutural e de modelos de mensuração. 2016, II 8 f. Dissertação (Mestrado em Administração) - Universidade de Brasilia. Brasília: UnB, 2016.

CUCHE, D. La notion de culture dans les sciences sociales. $\mathrm{Pa}$ ris: La Decouverte, 1996.

CROSBY, P. B. Liderança: a arte de tornar-se um executivo. São Paulo: Makron Books, 1991.

DEMO, G. et al. (2015). Políticas e Práticas de Gestão de Pessoas: Revisão Bibliométrica da Produção Nacional em Periódicos de Primeira Linha e Institucionalização da Pesquisa no Brasil entre 2010 e 20।4. In: Encontro de Gestão de Pessoas e Relações de Trabalho, 5 , 2015, Salvador. Anais... Salvador: EnGPR, 2015.

DEMO, G. Políticas e práticas de gestão de pessoas: possibilidades de diagnóstico para gestão organizacional. In MENDONÇA, H.; FERREIRA, M. C.; NEIVA, E. R. (Org.). Análise e Diagnóstico organizacional: Teoria e Prática, $\mathrm{p}$. I 17- | 47. São Paulo:Vetor, 2016.

DEMO, G. et al. Human Resources Management Policies and Practices Scale (HRMPPS): exploratory and confirmatory factor analysis. Brazilian Administration Review, v. 9, n. 4, 395-420, 2012.

DEMO, G. et al. Políticas e práticas de recursos humanos. In SIQUEIRA, M. M. M. (Org.). Novas Medidas do Comportamento Organizacional: Ferramentas de Diagnóstico e de Gestão, p. 240-245. Porto Alegre: Artmed, 2014.

DEMO, G.; ROZZETT, K. Human Resource Management Policies and Practices (HRMPP): scale validation in the United States. Inter- 
national Journal of Strategic Management, v. 12, n. 3, p. 4I66, 2012.

DESSLER, G. Human resource management. 9th ed. New Jersey: Prentice Hall, 2002.

DEVANNA, M. A.; FOMBRUN, C. J.; TICHY, N. M. A framework for strategic human resource management. In FOMBRUN, C. J.; TICHY, M. M.; DEVANNA, M. A. (Eds.) Strategic Human Resource Management, p. 33-5I. New York: John Wiley, 1984.

DIETZ, G.; WILKINSON, A.; REDMAN, T. Involvement and participation. In WILKINSON, A. et al. (Eds.). The SAGE handbook of human resource management, p. 245-268. London: Sage, 2010.

EMÍLIO, E.V.; MARTINS, M. C. F. Resiliência e autoconceito profissional em policiais militares: um estudo descritivo. Mudanças - Psicologia da Saúde, v. 20, n. I-2, p. 23-29, 2012.

FERNANDES, M. E. R.; MARQUES, A. L.; CARRIERI, A. P. Identidade organizacional e os componentes do processo identificatório: uma proposta de integração. Cadernos EBAPE.BR, v. 7, n. 4, p. 688703, 2009.

FERNANDES, K. R; ZANELLI, J. C. O processo de construção e reconstrução das identidades dos indivíduos nas organizações. Revista de Administração Contemporânea, v. 10, n. I, p. 55-72, 2006.

FIELD, A. Descobrindo a estatística usando o SPSS. Porto Alegre:Artmed, 2009.

FIORELLI, J. O. Psicologia para Administradores: integrando teoria e prática. 3. ed. São Paulo: Atlas, 2000.

FISCHER, A. L. (1998). A constituição do modelo competitivo de gestão de pessoas no
Brasil: um estudo sobre as organizações consideradas exemplares. 1998, 393 f. Tese (Doutorado em Administração) - Universidade de São Paulo. São Paulo: USP, 1998.

GOMIDE, S. Jr.; SILVESTRIN, L. H. B.; OLIVEIRA, A. de F. Bem-estar no trabalho: o impacto das satisfações com os suportes organizacionais e o papel mediador da resiliência no trabalho. Revista Psicologia: Organizações e Trabalho, v. I5, n. I, p. 19-29, 2015.

GUEST, D. Human resource management and industrial relations. The Journal of Management Studies, v. 24, n. 5, p. 503-52I, 1987.

HAIR, J. F. Jr. et al. Análise multivariada de dados. 6 a ed. Porto Alegre: Bookman, 2009.

HATCH, M. J.; SCHULTZ, M. The dynamics of organizational identity. Human Relations, v. 55, n. 8, p. 899-929, 2002.

HORTA, P.; DEMO, G.; ROURE, P. Políticas de Gestão de Pessoas, confiança e bem-estar no trabaIho: estudo em uma multinacional. Revista de Administração Contemporânea, v. 16, n. 4, 566$585,2012$.

HOYLE, R. H. Structural equation modeling: concepts, issues, and applications. Thousand Oaks, CA: Sage Publications, 1995.

JOB, F. P. P. Resiliência na organização: estudo de caso da medição e avaliação da resiliência de indivíduos em uma organização industrial. Revista Faculdade de Ciências Médicas de Sorocaba, v. 5 , n. I, p. 33-42, 2003.

LEGGE, K. Human resource management: rethorics and realities. London: Macmillan, 1995.

LEGGE, K. Human resource management. In ACKROYD, S. et al. (Eds.). The Oxford handbook of work and organization.
Oxford, UK: Oxford University Press, 2006.

LITTLE, L. M.; SIMMONS, B. L.; NELSON, D. L. Health among leaders: positive and negative affect, engagement and burnout, forgiveness and revenge. Journal of Management Studies, v. 44, n. 2, p. 243-260, 2007.

MACHADO, H.V.A identidade e o Contexto Organizacional: Perspectivas de Análise. Revista de Administração Contemporânea, v. 7, n. especial, p. 5I-73, 2003.

MACHADO-DA-SILVA, C. L.; NOGUEIRA, E. E. Identidade organizacional: um caso de manutenção, outro de mudança. Revista de Administração Contemporânea, v. 5, n. especial, p. 35-58, 200 I.

MAEL, F. A. Organizational identification: construct redefinition and a field application with organizational alumni. 1988, 308 f. Tese (Doutorado) Wayne State University. Detroit: WSU, 1988.

MAEL, F. A.; ASHFORT, B. E. Alumni and their alma mater: a partial test of the reformulated model of organizational identification. Journal of Organizational Behavior, v. 13, n. 2, p. 103-123, 1992.

MARÔCO, J. Análise de equações estruturais: Fundamentos teóricos, software e aplicações. Lisboa: Report Number, 2010.

MASCARENHAS, A. O.; KIRSCHBAUM, C. Fundamentos de gestão estratégica de pessoas. In MASCARENHAS, A. O. (Org). Gestão estratégica de pessoas: evolução, teoria e crítica, p. 23-30. São Paulo: Cengage Learning, 2008.

MATHIS, R. L.; JACKSON, J. H. Human resource management. IOth ed. Ohio: South- Western/ Thomson, 2003.

MELO, E. A. A. Escala de avaliação do 
estilo gerencial (EAEG): desenvolvimento e validação. Revista Psicologia: Organizações e Trabalho, v. 4, n. 2, p. 3I-62, 2004. MOINGEON, B.; RAMANANTSOA,

B. Understanding corporate identity: the French school of thought. European Journal of Marketing, v. 3I, n. 5, p. 383-395, 1997.

MORRIS, S.; SNELL, S. The evolution of HR strategy: adaptations to increasing global complexity. In WILKINSON, A. et al. (Eds.). The SAGE handbook of human resource management, $p$. 8499. London: Sage, 2010.

MUCKINSKY, P. M. Psicologia organizacional. São Paulo: Pioneira Thomson Learning, 2004.

MYERS, R. H. Classical and modern regression with applications. Boston: PWS-Kent Publishing Company, 1990.

NAG, R.; CORLEY, K.; GIOIA, D. The Intersection of Organizational Identity, Knowledge, and Practice: Attempting Strategic Change via Knowledge Grafting. The Academy of Management Journal, v. 50, n. 4, p. 82I-847, 2007.

NASCIMENTO, T. G. Desempenho profissional: relações com valores, práticas e identidade no serviço policial. 2014, $577 \mathrm{f}$. Tese (Doutorado em Administração) - Universidade de Brasília. Brasília: UnB, 2014.

NASCIMENTO, T. G. et al. Identidade no trabalho e a influência de aspectos sociodemográficos: um estudo da diferença entre grupos de policiais militares do Distrito Federal. Revista Brasileira de Segurança Pública, v. 2, n. 7, p. 90-117, 2013.

PASQUALI, L. (2010). Instrumenta- ção psicológica: fundamentos e práticas. Porto Alegre:Artmed. PAZ, M. G.T. Poder e saúde organizacional. In TAMAYO, A. (Org.). Cultura e saúde nas organizações, p. 127-I54. Porto Alegre: Artmed, 2004.

PESCE, R. P. et al. Adaptação transcultural, confiabilidade e validade da escala de resiliência. Cadernos de Saúde Pública, v. 2I, n. 2, p. 436-448, 2005.

PRATI, L. M.; MCMILLAN-CAPEHART, A.; KARRIKER, J. H. Affecting organizational identity: A manager's influence. Journal of Leadership \& Organizational Studies, v. 15, n. 4, p. 404-415, 2009.

PRATT, M. G; FOREMAN, P. O. The beauty of and barriers to organizational theories of identity. Academy of Management Review, v. 25, n. I, p. I 4I-I 43, 2000.

RAYKOV, T.; MARCOULIDES, G. A. A first course in structural equation modeling. 2nd ed. Mahwah, NJ: Lawrence Erlbaum Associates, 2006.

RIBEIRO, A. C. A. et al. Resiliência no trabalho contemporâneo: promoção e/ou desgaste da saúde mental. Psicologia em Estudo, v. 16, n. 4, p. 623-633, 2011.

RIKETTA, M. Organizational identification: A meta-analysis. Journal of Vocational Behavior, v. 66, n. 2, p. 358-384, 2005.

SCHULTZ, M.; HERNES, T. A Temporal Perspective on Organizational Identity. Organization Science, v. 24, n. I, p. I-2I, 2013.

SCHUMACKER, R. E., \& LOMAX, R. G. A beginner's guide to structural equation modeling. Mahwah, NJ: Lawrence Erl- baum Associates, 2004.

SIQUEIRA, M. M. M. Envolvimento com o trabalho. In SIQUEIRA, M. M. M. (Org.). Medidas do comportamento organizacional: ferramentas de diagnóstico e de gestão, p. 139-143. Porto Alegre:Artmed, 2008.

SISSON, K. Personnel management: paradigms, practice and prospects. In SISSON, K. (Ed.). Personnel management. 2nd ed., p. 3-50. Oxford, UK: Blackwell, 1994.

SOUSA, I. F; MENDONÇA, H. Burnout em professores universitários: impacto de percepções de justiça e comprometimento afetivo. Psicologia:Teoria e Pesquisa, v. 25, n. 4, p. 499-508, 2009.

TABACHNICK, B. G.; FIDELL, L. S. Using Multivariate Statistics. 6th ed. Boston: Pearson, 2013.

TATAW, D. Toward human resource management in inter-professional health practice: linking organizational culture, group identity and individual autonomy. International Journal of Health Planning and Management, v. 27, n. 2, p. I30-I49, 2012.

TYLER, T. R. Why people cooperate with organizations: An identity -based perspective. Research in Organizational Behavior, v. 2I, p. 20I-246, 1999.

ULRICH, D. et al. Employee and customer attachment: synergies for competitive. Human Resource Planning, v. 14, n. 2, p. 89-102, 1991.

WOOD,T.Jr.;TONELLI,M.J.; COOKE, B. Colonização e neo-colonização da gestão de recursos humanos no Brasil (1950-2010). Revista de Administração de Empresas, v. 5I, n. 3, p. 232-243, 201 I. 